\title{
The B00 Model Coil in the ATLAS Magnet Test Facility
}

\author{
A. V. Dudarev, E. W. Boxman, H. H. J. ten Kate, O. P. Anashkin, V. E. Keilin, and V. V. Lysenko
}

\begin{abstract}
A 1-m size model coil has been developed to investigate the transport properties of the three aluminumstabilized superconductors used in the ATLAS magnets. The coil, named B00, is also used for debugging the cryogenic, power and control systems of the ATLAS Magnet Test Facility. The coil comprises two double pancakes made of the Barrel Toroid and End-Cap Toroid conductors and a single pancake made of the Central Solenoid conductor. The pancakes are placed inside an aluminum coil casing. The coil construction and cooling conditions are quite similar to the final design of the ATLAS magnets. The $\mathrm{BO0}$ coil is well equipped with various sensors to measure thermal and electro-dynamic properties of the conductor inside the coils. Special attention has been paid on the study of the current diffusion process and the normal zone propagation in the ATLAS conductors and windings. Special pick-up coils have been made to measure the diffusion at different currents and magnetic field values.
\end{abstract}

Index Terms - superconducting cables, test facilities, superconducting magnets, magnetic fields.

\section{INTRODUCTION}

$\mathrm{T}$ The ATLAS Magnet Test Facility where the individual ATLAS magnets [1]-[3] will be tested in the next three years has been built at CERN and is now in the commissioning stage. All subsystems for cryogenics, power, vacuum and magnet control have been tested separately to verify their functional parameters. The cryogenic system is able to provide about $80 \mathrm{~g} / \mathrm{s}$ of liquid helium flow through long transfer lines to the indirectly cooled magnets. The $24 \mathrm{kA} / 6 \mathrm{~V}$ power supply with distribution busbar system is installed. A control and safety system is built to acquire information from a few hundred different sensors and to protect the coils.

The first large object that is planned to be tested in the test facility is a 9-m long prototype of the ATLAS Barrel Toroid

Manuscript received September 16, 2000. This work was supported by ATLAS Collaboration under Grant No. BS123456

A. V. Dudarev is with CERN, Geneva 23, CH-1211, Switzerland (telephone: 41-22-767-1134, e-mail: Alexey.Dudarev@cern.ch).

E.W. Boxman is with CERN, Geneva 23, $\mathrm{CH}-1211$, Switzerland (telephone: 41-22-767-8108, e-mail: Hubert.Boxman@cern.ch).

H. H.J. ten Kate is with CERN, Geneva 23, CH-1211, Switzerland (telephone: 41-22-767-1187, e-mail: Herman.tenKate@cern.ch).

O. P. Anashkin is with RRC "Kurchatov Institute", 123128, Kurchatov sq. Moscow, Russia (telephone: 7-095-196-9702, e-mail: ana@isssph.kiae.n).

V.E. Keilin is with RRC "Kurchatov Institute", 123128, Kurchatov sq. Moscow, Russia (telephone: 7-095-196-9911, e-mail: kev@isssph.kiae.ru).

V. V. Lysenko is with RRC "Kurchatov Institute", 123128, Kurchatov sq., Moscow, Russia (telephone: 7-095-196-9702, e-mail: lysenko@isssph.kiae.ru). coil [4]. Although it is not a full-size coil, its stored energy is about $50 \mathrm{MJ}$, which makes this coil unacceptable for debugging the new test station. In 1999, it was decided to build a 1-m size magnet named B00 for setting-up and commissioning of the test facility. This coil has been constructed by RRC "Kurchatov Institute" and is being tested at CERN.

The second purpose of the B00 model coil is to investigate in detail the transport properties of the ATLAS aluminumstabilized superconducting cables in conditions very close to the operating parameters. Three aluminum-stabilized superconductors have been used in the windings. Two double pancakes are made of the Barrel Toroid (BT) and End-Cap Toroid (ECT) conductors and a single pancake is made of the Central Solenoid (CS) conductor. The pancakes are glued inside an aluminum coil casing. The casing has an indirect cooling similar to the cooling conditions of the ATLAS magnets.

\section{B00 DESIGN}

\section{A. Conductors and Winding}

The design of the model coil is based on an assumption that the coil will be used to study stability, normal zone propagation, ac losses and current diffusion. A quench will be induced many times by heaters. This means that the stored energy of a coil has to be minimized to avoid long recovery time after a quench. At the same time, the magnetic field on the conductor itself has to be not far from the nominal value of the ATLAS magnet design to provide the proper conditions for the conductor study. The ATLAS conductors are superconducting NbTi Rutherford cables embedded in a high purity aluminum cladding by extrusion (see Table I).

Only the BT conductor used in the model coil is not the final one as the number of strands in the cable is slightly increased.

TABLEI

BOO CONDUCTOR PROPERTIES

\begin{tabular}{lllll}
\hline \multicolumn{1}{c}{ Conductor } & \multicolumn{2}{c}{ BT } & ECT & CS \\
& & & & \\
\hline Dimensions & $\mathrm{mm}$ & $57 \times 12$ & $41 \times 12$ & $30 \times 4.25$ \\
Strand diameter & $\mathrm{mm}$ & 1.3 & 1.3 & 1.22 \\
Number of strands & & $\begin{array}{l}32(38 \mathrm{in} \\
\text { final cable })\end{array}$ & 40 & 12 \\
Al:Cu ratio & & $36.4: 1$ & $24.7: 1$ & $14: 1$ \\
Critical current at 5 T\& & $\mathrm{kA}$ & $>47$ & $>55$ & $>20$ \\
$4.5 \mathrm{~K}$ & & & & \\
Operating current & $\mathrm{kA}$ & 20 & 20 & 7.6 \\
RRR at 1 T \& 4.5 K & & $>400$ & $>400$ & $>250$ \\
Stabilizer area & $\mathrm{mm}^{2}$ & 630 & 430 & 110 \\
\hline \hline
\end{tabular}


TABLE I

MAIN PARAMETERS OF COILS

\begin{tabular}{|c|c|c|c|c|}
\hline & & BT & ECT & $\mathrm{CS}$ \\
\hline Type of winding & & $\begin{array}{l}\text { Double } \\
\text { pancake }\end{array}$ & $\begin{array}{l}\text { Double } \\
\text { Pancake }\end{array}$ & $\begin{array}{l}\text { Single } \\
\text { Pancakt }\end{array}$ \\
\hline Inner dianneter & $\mathrm{mm}$ & 360 & 360 & 236 \\
\hline Outer diameter & $\mathrm{mm}$ & 616 & 610 & 290 \\
\hline Axial length & min & 116.1 & 83 & 30.2 \\
\hline Number of turns & & 20 & 20 & 6 \\
\hline Length of the conductor & $\mathrm{m}$ & 31 & 31 & 5 \\
\hline Self inductance & $\mathrm{mH}$ & 0.21 & 0.22 & 0.018 \\
\hline $\begin{array}{l}\text { Peak field \& } 20 \mathrm{kA} \text { in } \mathrm{BT} \\
+ \text { ECT coils }\end{array}$ & $\mathrm{T}$ & 2.8 & 2.8 & - \\
\hline Stored energy & $\mathrm{kJ}$ & & 120 & \\
\hline $\begin{array}{l}\text { Peak field \& } 20 \mathrm{kA} \text { in BT } \\
+\mathrm{ECT}+\mathrm{CS} \text { coils }\end{array}$ & $\mathrm{T}$ & 2.8 & 2.8 & 4.9 \\
\hline Stored energy & $\mathrm{kJ}$ & & 135 & \\
\hline
\end{tabular}

Because of the exceptional dimensions of the ATLAS toroidal magnets, the dimensions of the conductor are also quite large for winding of a small coil. The circular geometry of the coils has been chosen to minimize the size of the magnet and to simplify the winding process. The coils are wound onto a G-10 cylindrical frame. For the ECT and BT double pancakes, the winding process started from the middle of the conductor. An isolating insert was placed inside the inner turn of the winding to provide a smooth layer-to-layer transition with the radius needed.

Due to the circular shape of the winding, it was possible to impregnate the coils with an epoxy resin during the winding procedure. The conductor surface was cleaned mechanically and chemically and a fiberglass insulation tape with the resin was wrapped around with $50 \%$ overlap (see Fig. 1). A $0.5 \mathrm{~mm}$ G10 insulation sheet is present between the pancakes. After winding and removing from the winding machine the coils were checked for shorts by ac measurements.

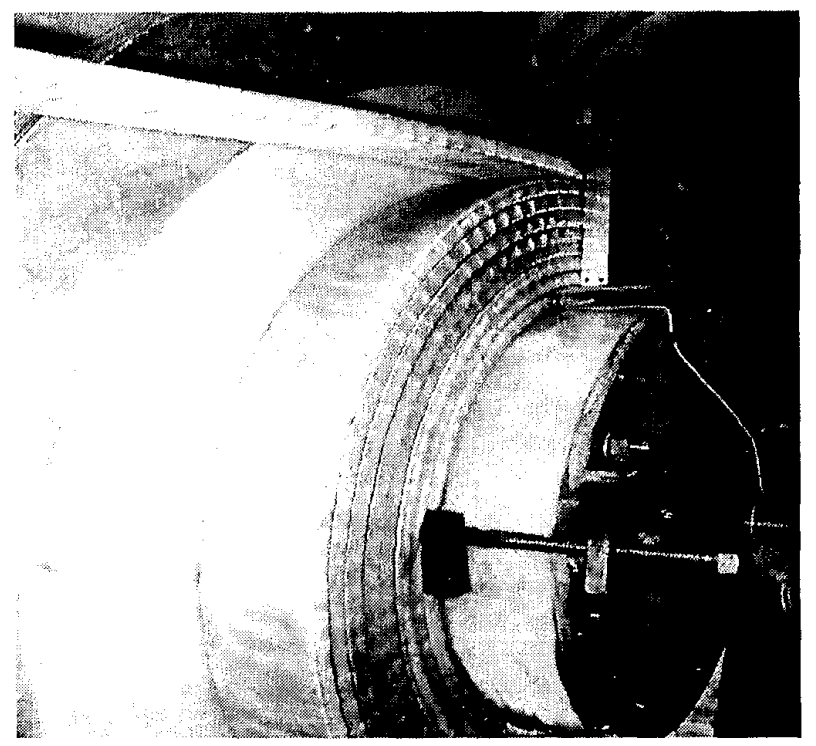

Fig. 1. Winding of the BT double pancake. The fiberglass tape with epoxy resin is wrapped with $50 \%$ overlap.

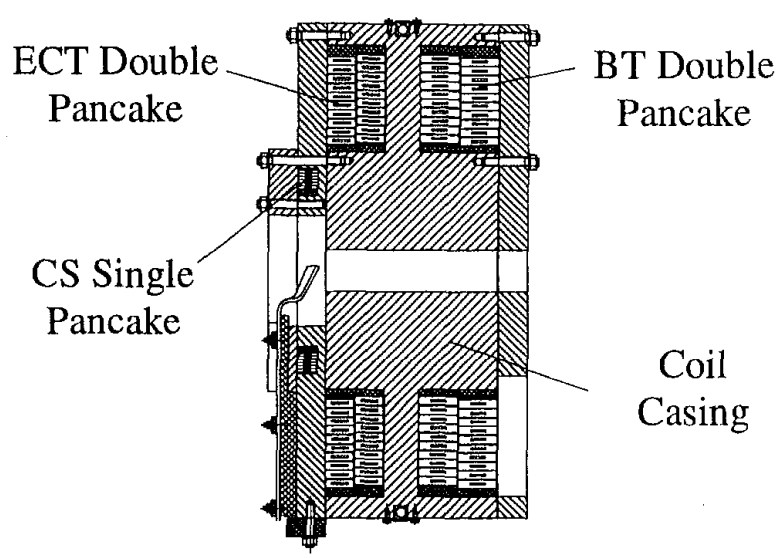

Fig. 2. The layout of the B00 model coil.

\section{B. Coils and Coil Casing}

The B00 main parameters are given in Table II and the layout of the coil is shown in Fig. 2. The ECT and BT double pancakes are placed and glued in a thick cylindrical casing made of aluminum alloy. Both sides of the coil casing are covered by aluminum plates. The CS single pancake is glued into one plate. A few $20 \mathrm{~mm}$ slots on each plate are machined for an easy access to the conductor where the different sensors are installed. Also the cover plates are not glued to the casing of the coils and can be removed. This gives the possibility to install additional diagnostic sensors when required.

The assembled coil casing is shown in Fig. 3. The magnet is cooled with liquid helium flowing inside an aluminum pipe that is glued into the groove in the casing body. The pipe itself and the way in which it is attached to the coil casing are similar to the final design of the Barrel and EndCap Toroids.

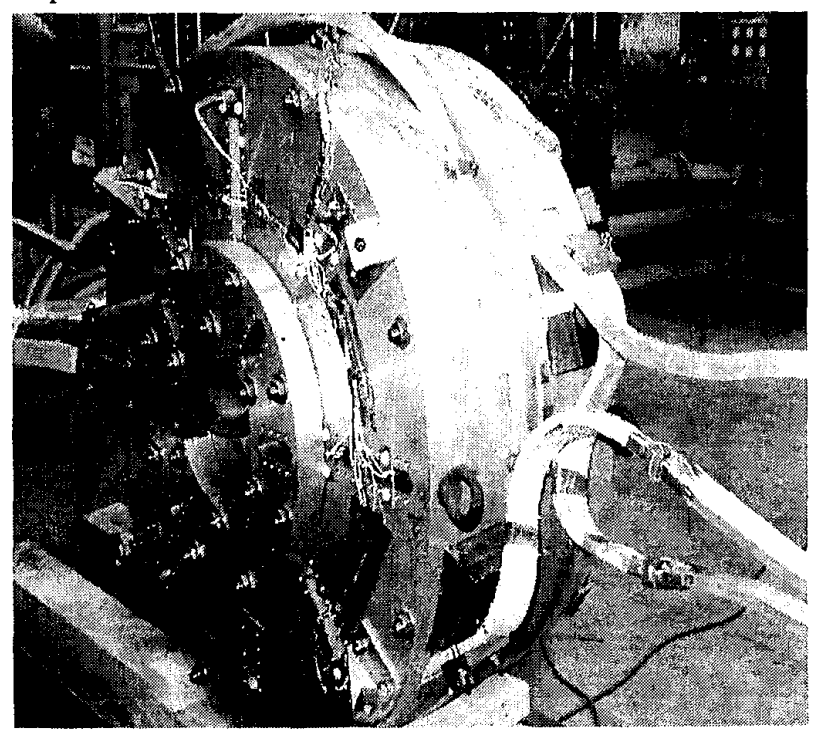

Fig. 3. The cold mass of the B00 model coil. 
The critical current of the CS conductor is much less than the critical current of the BT or ECT conductors. So it is planed to test the BT and ECT double pancakes without the $\mathrm{CS}$ conductor at the high current up to $24 \mathrm{kA}$ and then to connect the CS pancake for its test.

\section{Cryostat}

The aluminum coil casing is suspended inside the outer stainless steel vessel with the help of eight stainless steel tierods (four upper and four lower). An aluminum thermal radiation shield is installed in-between the magnet and the outer vessel and is supported with second set of stainless steel tie-rods. The screen is thermally connected to the aluminum pipe cooled with $60 \mathrm{~K}$ helium gas. The outer surface of the screen is covered with multilayer insulation. The design of the cryostat is done in such a way that the coil can be removed easily from the vacuum vessel for the installation of diagnostics without touching the radiation shield. The calculated head load is about $0.5 \mathrm{~W}$ to the cold mass and about $30 \mathrm{~W}$ to the radiation shield. The cooling down time of the $350-\mathrm{kg}$ cold mass is expected to be about 24 hours.

\section{B00 COIL AT TEST FACILITY}

\section{A. Service Turrets}

The 9-m prototype and the final ATLAS Barrel Toroid coils require a special interface to the power and cryogenic systems. Two service turrets have to be connected to the coils. The same turrets are used here and have to be checked during the test of the B00 model coil (see Fig. 4). 24-kA current leads are installed in the upper turret named CLT.

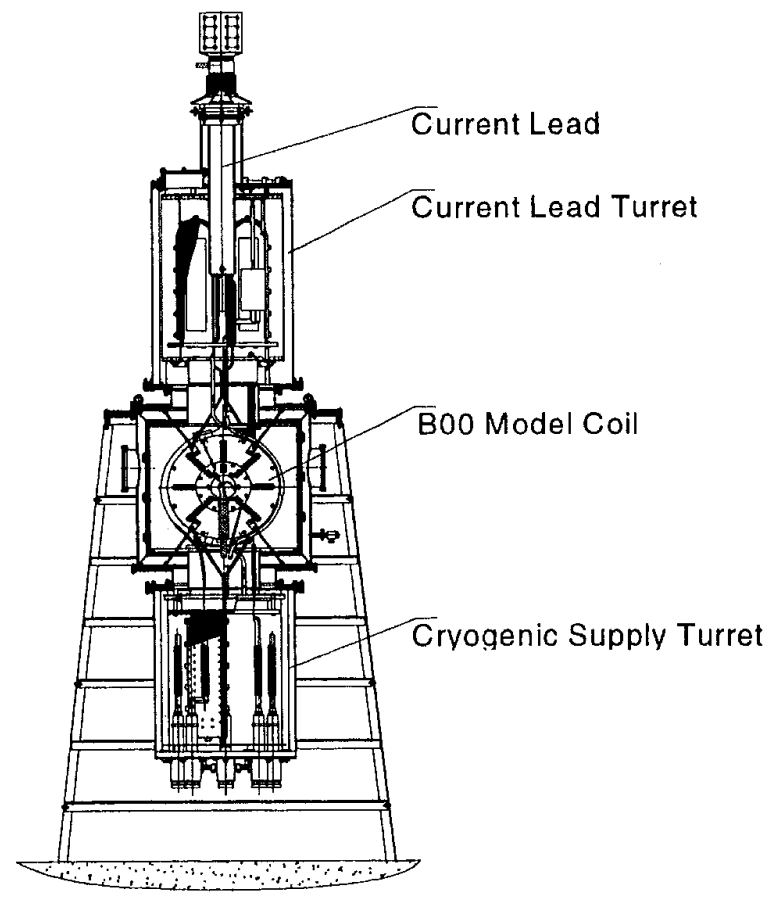

Fig. 4. Test setup: the B00 model coil with the service turrets.

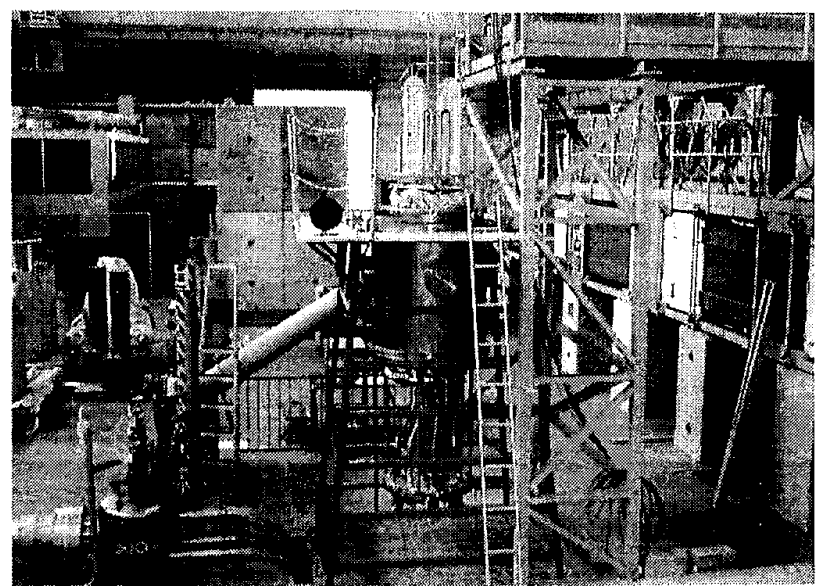

Fig. 5. B00 model coil at the ATLAS Magnet Test Facility.

In the bottom turret, all cryogenic connections are placed. The helium inlet and outlet pipes are connected to bayonets where the flexible feeding lines of the cryogenic plant are mounted. In addition, a welded coil-to-coil joint with its cooling block is suited in this cryogenic supply turret (CST). The CST has been tested to define the maximum heat load in the joint. It was found that a joint with resistance of $5 \mathrm{n} \Omega$ is acceptable. The B00 coil at the final stages of assembly is shown in Fig. 5.

\section{B. Instrumentation of BOO Coil}

The B00 model coil is well equipped with various sensors to study thermal and electrical properties of the ATLAS conductors and the thermal behavior of the cold mass during the cooling process. More than seventy sensors are installed on the coils and the coil casing in order to characterize the three different aluminum-stabilized superconductors of the ALTAS magnet system. Normal zone propagation, current diffusion and recovery process into the stabilizer, stability and ramp losses are to be measured during the tests of the coil. The layout of the sensors placed on the BT double pancake is shown in Fig. 6. The ECT coil is equipped with similar diagnostics.

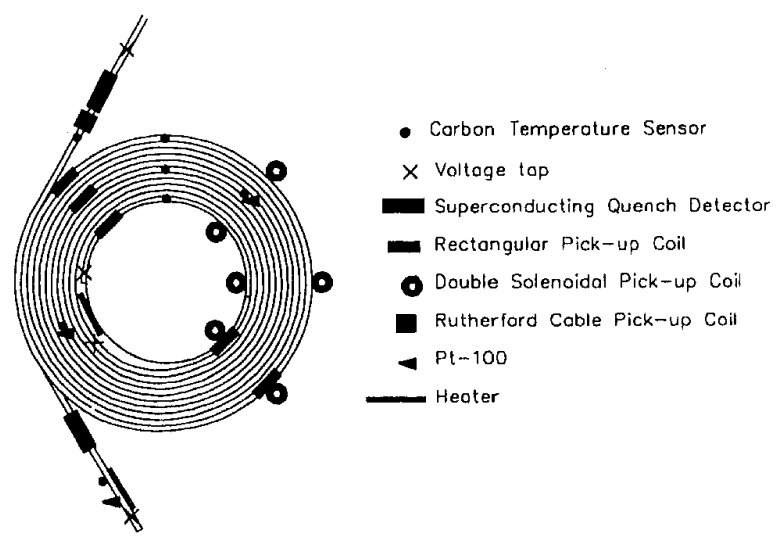

Fig. 6. The instrumentation the BT double pancake. 

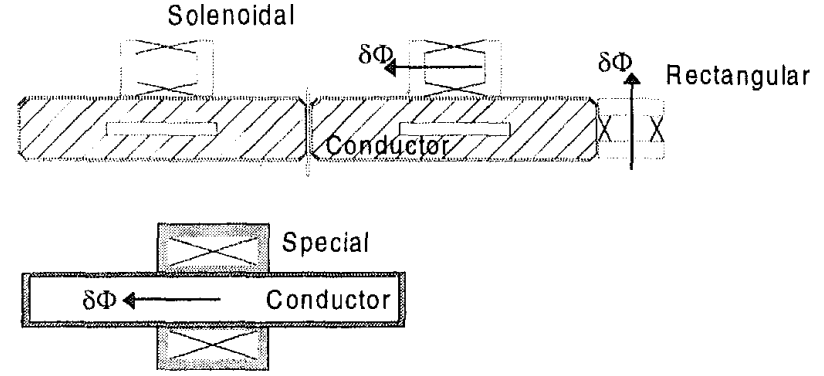

Fig. 7. The pick-up coils.

The overall performance of the coil during normal operation is to be tested. The coil casing consist out of an industrial Aluminum alloy, which has a poor heat conductivity compared to pure metals. Therefore, special attention will be made to investigate the indirect cooling capacity of the system with calibrated temperature sensors. During this test, an attempt will be made to measure the ac losses induced by ramping up or down the current.

One of the interesting processes between the superconducting state and the normal state is the current diffusion effect into the stabilizing cladding [5],[6]. Due to the redistribution of the current, the magnetic field changes around the conductor. The process is rather fast and fast data acquisition is needed, which requires high signal/noise ratio. A way to measure this process is voltage measurements on small pick-up coils positioned directly on the conductor. Three types of pick-up coils are made with typical 2000 turns on a G10 spool. The wire diameter is $0.06 \mathrm{~mm}$. A double solenoidal pickup coils are built to measure the flux change on a wide side of the conductor. They are placed inside the coil casing close to the conductor as depicted in Fig. 7. The rectangular pick-up coils are placed next to the conductor in the slots of the cover plates. Pickup coils are electrically isolated from the conductor. Due to the high number of turns proper signals are expected that simplifies the fast data acquisition system.

Another important property is the normal zone propagation. For safety reasons, the power supply has an electrical earth. Together with the 50-m long busbar, a rather high common mode resistance ration is expected during direct voltage measurement on the coil. Also the resistance of the conductor in the normal state is very low due to high purity aluminum, which results in low voltage over the conductor during a quench. It is quite difficult to acquire low voltage across the normal zone with a fast data acquisition system.
For these reasons, the quench propagation measurement with voltage taps will be a challenge and the pick-up coils are used to measure the normal zone propagation.

In the beginning of the current diffusion, the current switches from the Rutherford cable with a solenoidal field component to the aluminum stabilizer. This process induces a small flux change in the direction along the conductor. A special pick-up coil is developed and placed around the conductor. In this way, it is expected to measure the front of the normal zone with high accuracy. During these test minimum propagation zone and minimum quench energy will be determined at different initial temperatures and at different currents. Finally, for redundancy and validation, superconducting quench detectors made of a thin superconducting NbTi wire are placed on the conductor.

\section{CONCLUSION}

The B00 model coil has been developed and built for debugging and commissioning of the ATLAS Magnet Test Facility. In addition the coil is well equipped with diagnostics to fully characterized the ATLAS conductor properties. The coil is in the final stages of preparations at CERN and will be tested in the last quarter of the year 2000 .

\section{ACKNOWLEDGMENT}

The authors wish to thank the team of engineers and technicians from RRC "Kurchatov Institute" for the manufacturing and assembly of the model coil. A special thank to F. Haug, O. Pirotte, J, Metselaar and all team members of the LHC/ECR group for their continuous support and their leading assistance in the installation work at CERN.

\section{REFERENCES}

[1] H.H.J. ten Kate, "The superconducting magnet system for the ATLAS detector at CERN," IEEE Trans.Appl.Supercond., vol. 10, No. 1, pp. 347-352, March 2000 .

[2] H.H.J. ten Kate, "The ATLAS superconducting magnet system," this conference.

[3] A. Dael et al, "Progress in the design of the Barrel Toroid magnet for the ATLAS experiment and associated R\&D at CEA-Saclay and INFNMilano," MT-15 Proceedings, part I, pp.92-95.

[4] P. Miele et al, "Status and commissioning of the ATLAS Magnet test facility at CERN," this conference.

[5] A.V.Dudarev, A.V.Gavrilin, H.H.J. ten Kate et all, "Quench propagation and protection analysis of the ATLAS Toroids," IEEE Trans.Appl.Supercond., vol. 10, No. 1, pp. 365-368, March 2000.

[6] E.W. Boxman, A.V. Dudarev, H.H.J. ten Kate, "Current diffusion in Aluminum stabilized superconducting cables", this conference 